\title{
Correlation Functions for Hubbard-Type Models: The Exact Results for the Gutzwiller Wave Function in One Dimension
}

\author{
F. Gebhard \\ Physik-Department, Technische Universität München, D-8046 Garching, Federal Republic of Germany \\ and \\ D. Vollhardt \\ Max-Planck-Institut für Physik und Astrophysik, D-8000 München 40, Federal Republic of Germany
}

(Received 13 April 1987)

\begin{abstract}
We report the analytic, approximation-free evaluation of four different correlation functions for interacting fermions on a one-dimensional lattice in terms of the Gutzwiller wave function.
\end{abstract}

PACS numbers: $71.28 .+\mathrm{d}, 75.10 . \mathrm{Jm}$

Narrow-band metals, ${ }^{1}$ heavy-fermion materials, ${ }^{2}$ and liquid ${ }^{3} \mathrm{He}^{3}$ are well-known examples of correlated Fermi systems with a strong short-range, repulsive interaction. In the presence of a lattice the latter is often modeled by an interaction of two fermions with opposite spin on the same lattice site. The addition of a kinetic energy for next-neighbor hopping leads to the Hubbard model. $^{4,5}$ For its investigation, variational methods have proven to be helpful. Gutzwiller constructed a particularly simple variational wave function, ${ }^{4,6}$

$$
\left|\psi_{\mathrm{G}}\right\rangle=\prod_{i}\left[1-(1-g) n_{i \uparrow} n_{i \downarrow}\right]\left|\psi_{0}\right\rangle,
$$

where $n_{i \sigma}$ are number operators for spins $\sigma$ at site $i$ and $\left|\psi_{0}\right\rangle$ is the wave function of the noninteracting Fermi system. The correlation factor in (1) reduces the number of doubly occupied sites of $\left|\psi_{0}\right\rangle$, i.e., controls local charge fluctuations. The correlation parameter $0 \leq g$ $\leq 1$ has to be determined variationally. In spite of the simplicity of $\left|\psi_{G}\right\rangle$ and the long history of the problem exact analytic evaluations of such expectation values in the thermodynamic limit could not be performed-not even in one dimension. Therefore perturbative techniques, ${ }^{7}$ Gutzwiller-type approximations, ${ }^{8}$ and numerical methods ${ }^{9-13}$ were employed.

Most recently Metzner and Vollhardt ${ }^{14}$ presented a new, analytically tractable approach to the problem which allows for an exact evaluation of the expectation value $\langle\mathcal{O}\rangle=\left\langle\psi_{\mathrm{G}}|\mathcal{O}| \psi_{\mathrm{G}}\right\rangle /\left\langle\psi_{\mathrm{G}} \mid \psi_{\mathrm{G}}\right\rangle$ of an operator $\mathcal{O}$ in terms of $\mid \psi_{\mathrm{G}}$ - at least in one dimension (the method itself is applicable to arbitrary dimensions). Differing from other approaches, ${ }^{4,6,7}$ its expectation values are expressed as power series in $1-g^{2}$, whose coefficients can be calculated to arbitrary order. For example, the onsite interaction $H_{I}=U \sum_{i} D_{i}, D_{i}=n_{i \uparrow} n_{i \downarrow}$, is obtained as

$$
\left\langle H_{I}\right\rangle=U L g^{2} \sum_{m=1}^{\infty}\left(g^{2}-1\right)^{m-1} c_{m},
$$

with

$$
c_{m}=[L(m-1) !]^{-1} \sum_{\mathbf{f}_{1}, \ldots, \mathbf{f}_{m}}\left\{D_{\mathbf{f}_{1}} \cdots D_{\mathbf{f}_{m}}\right\}_{0}^{c},
$$

and $L$ the number of lattice sites. Here $\{\ldots\}_{0}^{c}$ implies the usual sum over all pairs of contractions in the noninteracting ground state (index zero) corresponding to connected graphs (superscript $c$ ) with, however, one essential difference: In these contractions the usual $\delta_{\mathbf{f}_{i}, \mathbf{f}_{j}}$ terms are always zero, since the application of Wick's theorem to $\left\langle D_{\mathbf{f}_{1}} \cdots D_{\mathbf{f}_{m}}\right\rangle_{0}\left(\langle\cdots\rangle_{0} \rightarrow\{\cdots\}_{0}\right)$ happened when all $\mathbf{f}_{i}$ were different. ${ }^{14}$ Nevertheless in the summation over the $\{\cdots\}_{0}$ this restriction on the $\mathbf{f}_{i}$ may be relaxed, allowing one to go over the connected graphs. The $c_{m}$ are then calculated as $c_{m}=(-n)^{m+1 / 2}(m+1)$, where $n=N / L \leq 1$ is the density of particles $\left(n_{\uparrow}=n_{!}\right.$ $\left.=\frac{1}{2} n\right) ;$ hence

$$
\left\langle H_{I}\right\rangle=\frac{1}{2} U L\left[g /\left(1-g^{2}\right)\right]^{2}\left(G^{2}-1-\ln G^{2}\right),
$$

with $G^{2}=1-\left(1-g^{2}\right) n$. This yields the density of doubly occupied sites $d=\left\langle H_{I}\right\rangle / U L$; for $1 \leq n \leq 2, d(n)$ $=d(2-n)+n-1$. The momentum distribution $\left\langle n_{k}\right\rangle$ has also been calculated-in this case by a recursive technique. Therefore the ground-state energy of a Hubbard Hamiltonian with general kinetic energy is obtained in terms of $\left|\psi_{\mathrm{G}}\right\rangle$ without approximation. ${ }^{14}$ The correlation parameter $g$ is then uniquely determined by the actual interaction $U$.

The approach may also be used to calculate correlation functions (CF's) in terms of $\left|\psi_{\mathrm{G}}\right\rangle$. Here we present approximation-free, analytic results for four different CF's in one dimension, which are relevant for Hubbardtype models. In such models a lattice site may be singly occupied, doubly occupied ( $D$ site), or empty (hole). We introduce number operators at site $i$ for the spin $\left(S_{i}^{z}\right.$ $\left.=n_{i \uparrow}-n_{i \downarrow}\right)$, density $\left(N_{i}=n_{i \uparrow}+n_{i \downarrow}\right), D$ sites $\left(D_{i}=n_{i \uparrow} n_{i \downarrow}\right)$, and holes $\left[H_{i}=\left(1-n_{i \dagger}\right)\left(1-n_{i \downarrow}\right)\right]$, and define the CF's between them as

$$
C_{j}^{X Y}=L^{-1} \sum_{i}\left\langle X_{i} Y_{i+j}\right\rangle-\langle X\rangle\langle Y\rangle,
$$

where $X_{i}, Y_{i}=S_{i}^{z}, N_{i}, D_{i}, H_{i}$ and $X=L^{-1} \sum_{i} X_{i}$, etc.; Fourier transforms are denoted by $C^{X Y}(\mathbf{q})$. For $n_{\uparrow}$ $=n_{\downarrow}=\frac{1}{2} n$ there are seven CF's, four of which are in- 
dependent.

The spin CF, $C_{j}^{S S}$, has already been studied in detail. $9,10,12,13$ For the Hubbard model exact values for $C_{j}^{S S}$ are only known for $j=1^{15}$ and $j=2^{16}$ in one dimension in the atomic limit with $n=1$, where the correspondence to the antiferromagnetic Heisenberg model may be employed. To evaluate $C^{S S}(\mathbf{q})$ we proceed as in the case of $\left\langle H_{I}\right\rangle$. We obtain

$$
C^{S S}(\mathbf{q})=2 \sum_{m=0}^{\infty}\left(g^{2}-1\right)^{m_{S}(m)}(\mathbf{q}),
$$

where

$$
\begin{aligned}
& S^{(m)}(\mathbf{q})=\frac{1}{L} \frac{1}{m !} \sum_{\mathbf{f}_{1}, \ldots, \mathbf{f}_{m+2}} e^{i \mathbf{q} \cdot\left(\mathbf{f}_{1}-\mathbf{f}_{2}\right)} \\
& \times\left\{\left[n_{\mathbf{f}_{1} \uparrow} n_{\mathbf{f}_{2} \uparrow}-n_{\mathbf{f}_{1} \uparrow} n_{\mathbf{f}_{2} \downarrow}\right] D_{\mathbf{f}_{3}} \cdots D_{\mathbf{f}_{m+2}}\right\}_{0}^{c} .
\end{aligned}
$$

The $s^{(m)}(\mathbf{q})$ again correspond to connected graphs, which join sites $\mathbf{f}_{1}$ and $\mathbf{f}_{2}$ across $m$ other lattice sites. In one dimension they are polynomials:

$$
s^{(m)}(q)=\sum_{l=0}^{m+1} a_{l}^{(m)}\left(\frac{1}{2} n\right)^{m+1-l}(|q| / \pi)^{l},
$$

with $|q| \leq 2 k_{\mathrm{F}}=\pi n$. The sum rule $(2 \pi)^{-1} \int \pi_{\pi} d q$ $\times C^{S S}(q)=n-2 d$ connects the $a_{l}^{(m)}$ with the coefficients $c_{m}$ which determine $d$ (see above). Use of particle-hole symmetry and the differentiability of $C^{S S}(q)$ with respect to $n$ at $n=1$ yields $a_{l}^{(m)}=\delta_{l, m+1}(-1)^{m / 2}(m+1)$. With the introduction of the function $F(x)=1-(1$ $\left.-g^{2}\right) x$, the spin CF is then found as $(Q=|q| / \pi)$

$$
C^{S S}(q)=-\frac{1}{1-g^{2}} \times \begin{cases}\ln F(Q), & 0 \leq Q \leq n, \\ \ln F(n), & n \leq Q \leq 1\end{cases}
$$

For $n=1, C^{S S}(q)$ diverges logarithmically at $|q|=2 k_{\mathrm{F}}$. In Fig. $1, C^{S S}(q)$ is shown for $n=0.7$ and different correlation parameters $g$ in comparison with the exact result for the noninteracting case. The numerical results

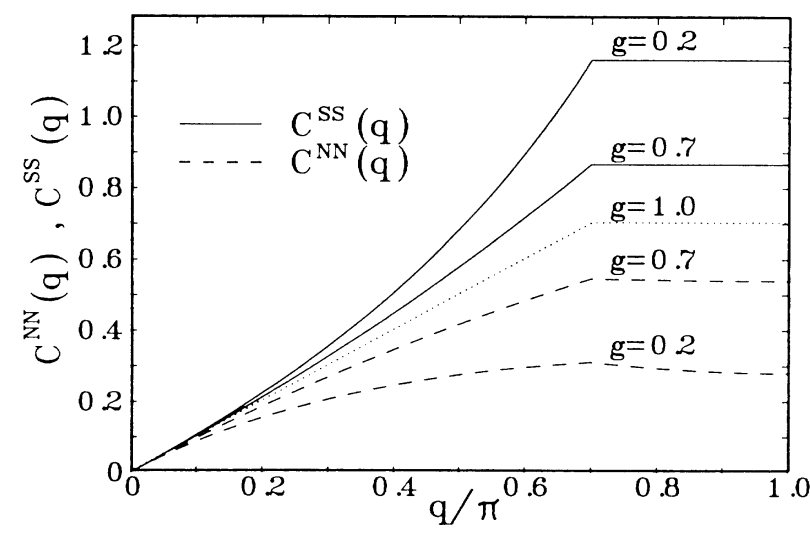

FIG. 1. The spin- and density-correlation functions $C^{S S, N N}(q)$ for $n=0.7$ and different correlation parameters $g$. In the noninteracting case $(g=1)$ they coincide.

for $C^{S S}(q)$ given by Yokoyama and Shiba ${ }^{13}$ agree extremely well with (4).

The expression for $C_{j}^{S S}$ in real space is easily calculated. In the atomic limit $(g=0)$ and for $n=1$,

$$
C_{j}^{S S}=\frac{\operatorname{Si}(\pi j)}{\pi} \frac{(-1)^{j}}{j}, j>0,
$$

where $\operatorname{Si}(x)$ is the sine integral. The numerical evaluations for $j=1^{9,12}$ and $j=2^{10}$ are therefore seen to be very accurate. The $(-1)^{j} / j$ behavior in (5), known from the exact result for the Hubbard model, ${ }^{17}$ was already suggested by Horsch and Kaplan. ${ }^{10}$ The corresponding divergence of $C^{S S}(q)$ at $|q|=2 k_{\mathrm{F}}$ (antiferromagnetic transition) reproduces the behavior of the exact result of the Hubbard model. In Table I we have compared the values from (5) with exact ${ }^{15,16}$ and numerical results for the antiferromagnetic Heisenberg chain. ${ }^{18,19}$ They are well described by (5) - both qualitatively (oscillation about $\left|C_{j}^{S S}\right| \rightarrow 1 / 2 j$ for $j \rightarrow \infty$ ) and

\begin{tabular}{|c|c|c|c|c|}
\hline \multirow{2}{*}{\multicolumn{2}{|c|}{$\begin{array}{c}\left.C_{j}^{S S}\right|_{\mathrm{GWF}, g}=0 \\
\text { Eq. (5) }\end{array}$}} & \multicolumn{3}{|c|}{$\left.C_{j}^{S S}\right|_{\mathrm{AHC}}$} \\
\hline & & Exact & Numerical $^{c}$ & Numerical $^{\mathrm{d}}$ \\
\hline 1 & -0.589490 & $-0.590863^{a}$ & $-0.59084(1)$ & $-0.5908(2)$ \\
\hline 2 & 0.225706 & $0.242719^{b}$ & $0.24260(2)$ & $0.24265(20)$ \\
\hline 3 & -0.177698 & & $-0.20047(8)$ & $-0.2009(1)$ \\
\hline 4 & 0.118742 & & $0.13798(8)$ & $0.1386(3)$ \\
\hline 5 & -0.104021 & & $-0.1211(4)$ & $-0.1235(3)$ \\
\hline 6 & 0.080534 & & $0.0943(6)$ & $0.0982(4)$ \\
\hline 7 & -0.073488 & & $-0.082(1)$ & $-0.0899(6)$ \\
\hline 8 & 0.060922 & & $0.069(1)$ & $0.0760(6)$ \\
\hline
\end{tabular}
quantitatively. On the other hand, for $n<1, C^{S S}(q)$ no

TABLE I. The spin-correlation function $C_{j}^{S S}$ in the atomic $(g=0)$ calculated with the Gutzwiller wave function (GWF), Eq. (5), as compared with exact and numerical results for the antiferromagnetic Heisenberg chain (AHC). Numbers in parentheses indicate uncertainties in the last digit. 
longer diverges at $q=2 k_{\mathrm{F}}$, but only shows a kink, in contrast to the numerical results. ${ }^{20}$

Next we calculate the density $\mathrm{CF}, C^{N N}$, the hole $\mathrm{CF}, C^{H H}$, and the CF between $D$ sites and holes, $C^{D H}$. They are related via a particle-hole transformation $(\bar{n}=2-n \leq 1)$ :

$$
C^{N N}(\mathbf{q}, n)=C^{H H}(\mathbf{q}, n)+C^{H H}(\mathbf{q}, \bar{n})-2 C^{D H}(\mathbf{q}, n), \quad C^{N N}(\mathbf{q}, n)=C^{N N}(\mathbf{q}, \bar{n}) .
$$

The density CF may be written as $C^{N N}(\mathbf{q})=C^{S S}(\mathbf{q})+T(\mathbf{q})$, where $T(\mathbf{q})=4 \sum_{m=0}^{\infty}\left(g^{2}-1\right)^{m} t^{(m)}(\mathbf{q})$ is determined by

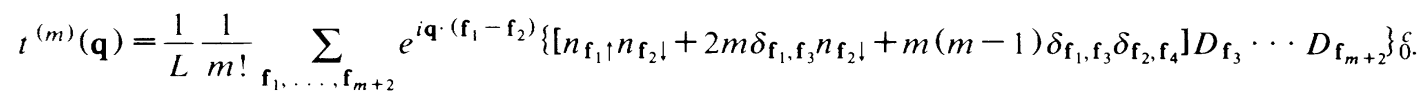

The same three terms in (7) ${ }^{21}$ also determine $C^{H H}$ and $C^{D H}$. In one dimension they all have the structure $n^{m+1} \sum_{l=0}^{m+1} b_{l}^{(m)} y^{l}$ with $y=|q| / \pi n$; in the third term $y$ is replaced by $2-y$ for $|q| \geq 2 k_{\mathrm{F}}$. There are then four distinct contributions, i.e., sets of coefficients $b_{l}{ }^{(m)}$, that have to be determined. To this end we employ (6) and the continuity of the first two derivatives of the above CF's at $n=1$. This yields just enough equations to calculate the coefficients.

For $\frac{2}{3} \leq n \leq 1$, the density $\mathrm{CF}$ is found as $(\bar{n}=2-n, \bar{Q}=2-Q)$

$$
C^{N N}(q)=\left\{\begin{array}{l}
Q\left[1-\frac{1}{2} \ln F(n-Q) / F(n)\right]=: C_{(\mathrm{i})}^{N N}(q), \quad 0 \leq Q \leq 2(1-n), \\
2(1-n)+\frac{g^{2}}{1-g^{2}} \ln \frac{F(\bar{n}-Q)}{F(n)}+\frac{1}{2} Q \ln \frac{F(\bar{n}-Q)}{F(n-Q)}=: C_{(\mathrm{ii})}^{N N}(q), \quad 2(1-n) \leq Q \leq n, \\
C_{(\mathrm{ii})}^{N N}(q)+\frac{1}{1-g^{2}} \ln F(\bar{n}-\bar{Q})+\frac{1}{2} Q \ln \frac{F(n-Q)}{F(\bar{n}-\bar{Q})}=: C_{(\mathrm{iii})}^{N N}(q), \quad n \leq Q \leq 1 .
\end{array}\right.
$$

For $n \leq \frac{2}{3}$, the boundaries $Q=2(1-n)$ and $Q=n$ of the three regimes (where the third and first derivatives by $q$, respectively, are discontinuous) have to be interchanged. In Fig. $1, C^{N N}(q)$ is shown for $n=0.7$ and different values of $g$. Again the numerical results of Ref. 13 are seen to be very accurate, and the suppression of density fluctuations is clearly borne out. In real space and for strong correlations $C_{j}^{N N}$ varies as $1 / j$ for $1<j \ll 1 / 2 d$, where $1 / 2 d$ is the average separation of $D$ sites and holes, and only for $j \gg 1 / 2 d \gg 1$ does it vanish as $1 / j^{2}$.

As pointed out by Gros, Joynt, and Rice ${ }^{12}$ the hole CF $C^{H H}(q)$ allows for a further assessment of the Gutzwiller wave function. For $n<1$ and $g=0$, holes act as noninteracting, spinless fermions. In this limit $C_{g}^{H H}=C_{0}=C_{g}^{N N}$.
Clearly, the exact result is given by the free density $\mathrm{CF}$ $C_{g=1}^{N N}$ with $n \rightarrow n_{h}=1-n, k_{\mathrm{F}} \rightarrow k_{\mathrm{F}}^{h}=\pi n_{h}$, i.e., $C_{g}^{H H}{ }_{0}(q)$ is equal to $|q| / 2 \pi$ for $|q| \leq 2 n_{h} / \pi$ and is equal to $n_{h}$ otherwise. ${ }^{12}$ There is a correlation hole of width $1 / 2 n_{h}$ around each empty site with oscillations of wavelength $1 / n_{h}$, the average separation of holes. In Fig. $2 C_{g}^{H H_{0}}(q)$, as obtained with $\left|\psi_{\mathrm{G}}\right\rangle$, is compared with the exact result. Although there is no sharp kink at $q=2 k_{\mathrm{F}}^{h}$, the overall feature is well borne out. The agreement improves for $n_{h} \rightarrow 0$. The kink at $q=2 k_{\mathrm{F}}$ is an artifact of the Gutzwiller wave function which is based on the ground state of free electrons rather than holes. ${ }^{12}$ The expression for $C^{H H}(q)$ for general $n \leq 1$ and $g$ has a similar structure as $C^{N N}(q)$. For $n=1$ one obtains

$$
C^{H H}(q)=\left[\frac{g^{2}}{2\left(1-g^{2}\right)}\right]^{2}\left[2-\left(\frac{2}{g^{2}}-1\right) C^{N N}(q)-C^{S S}(q)-\frac{2}{1-g^{2}} \frac{\pi}{q}\left(\frac{1}{g^{2}} C^{N N}(q)-C^{S S}(q)\right)\right]+d \frac{1+g^{2}}{1-g^{2}} \text {. }
$$

Finally, the CF between $D$ sites and holes is given by $(n=1)$

$$
C^{D H}(q)=C^{H H}(q)-\frac{1}{2} C^{N N}(q) .
$$

In real space and for strong correlations (10) yields $C_{j}^{D H} \simeq-\frac{1}{2} C_{j}^{N N}$. Hence, in the Gutzwiller wave function the correlation between $D$ sites and holes is essentially determined by the average separation of spins. On the other hand, for $g \rightarrow 0$ one should expect a strong nextneighbor correlation between $D$ sites and holes, since this makes it easier for an energetically costly $D$ site, once formed, to dissociate again. The missing correlation was first discussed by Kaplan, Horsch, and Fuble ${ }^{9}$ in their investigations of finite rings. ${ }^{22}$ Indeed, it may well be the main origin for the logarithmic corrections to $E$ in the approximation-free result obtained with $\left|\psi_{\mathrm{G}}\right\rangle{ }^{14}$ which make $E$ quite a bit larger than the exact result. ${ }^{23}$

The above results for the CF's in terms of $\left|\psi_{\mathrm{G}}\right\rangle$, together with the results for the momentum distribution $\left\langle n_{k}\right\rangle,{ }^{14}$ can be directly used to calculate the ground-state energy of Hubbard-type models with interactions more complicated than on-site interactions.

In summary, the correlation functions between up and 


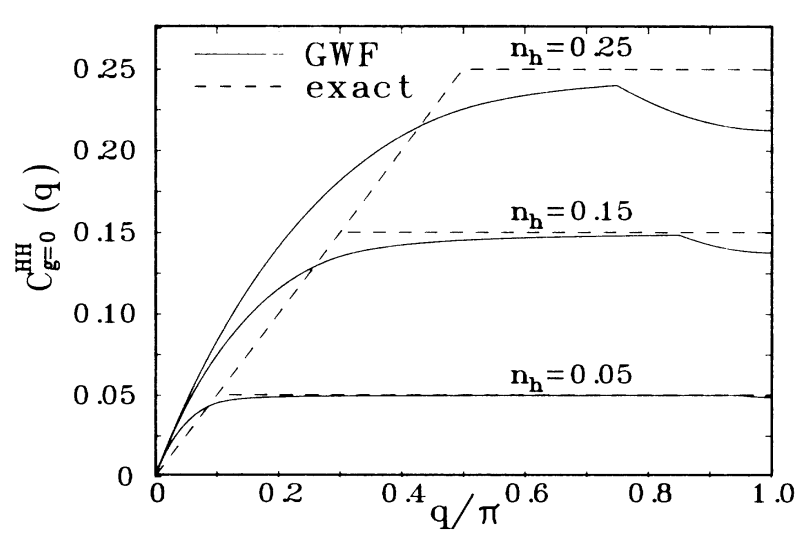

FIG. 2. The hole-correlation function in the atomic limit ( $g=0$ ) for different hole concentrations $n_{h}$ as compared with the exact result.

down spins, doubly occupied sites, and empty sites relevant for Hubbard-type models have been calculated analytically with the Gutzwiller wave function in one dimension for arbitrary density and correlation strength. Spin-spin correlations for $n=1$ and hole-hole correlations for $n \lesssim 1$ are in very good agreement with the available exact results in the atomic limit. On the other hand, as noted before, ${ }^{9}$ the correlations between doubly occupied sites and holes close to this limit appear insufficient. The results allow for a detailed, approximation-free evaluation of the quality of this variational wave function. In more than one dimension results may be obtained by numerical techniques.

We thank W. Metzner for valuable discussions.

\footnotetext{
${ }^{1}$ Electron Correlation and Magnetism in Narrow-Band Systems, edited by T. Moriya, Springer Series in Solid-State Sciences Vol. 29 (Springer-Verlag, Berlin, 1981).
}

${ }^{2}$ P. A. Lee, T. M. Rice, J. W. Serene, L. J. Sham, and J. W. Wilkins, Comments Condens. Matter. Phys. 12, 99 (1986).

${ }^{3}$ C. E. Campbell, in Progress in Liquid Physics, edited by C. A. Croxton (Wiley, New York, 1978).

${ }^{4}$ M. C. Gutzwiller, Phys. Rev. Lett. 10, 159 (1963).

${ }^{5}$ J. Hubbard, Proc. Roy. Soc. London A 276, 238 (1963).

${ }^{6}$ M. C. Gutzwiller, Phys. Rev. 134, A923 (1964), and 137, A1726 (1965).

${ }^{7}$ P. Horsch, Phys. Rev. B 24, 7351 (1981); D. Baeriswyl and K. Maki, Phys. Rev. B 31, 6633 (1985).

${ }^{8}$ For a review, see D. Vollhardt, Rev. Mod. Phys. 56, 99 (1984).

${ }^{9}$ T. A. Kaplan, P. Horsch, and P. Fulde, Phys. Rev. Lett. 49, 889 (1982)

${ }^{10}$ P. Horsch and T. A. Kaplan, J. Phys. C 16, L1203 (1983).

${ }^{11}$ K. Hashimoto, Phys. Rev. B 31, 7368 (1985).

${ }^{12}$ C. Gros, R. Joynt, and T. M. Rice, Phys. Rev. B 36, 381 (1987).

${ }^{13}$ H. Yokoyama and H. Shiba, Institute for Solid State Physics Technical Report No. 1729, Ser. A, 1986 (unpublished).

${ }^{14} \mathrm{~W}$. Metzner and D. Vollhardt, Max-Planck-Institut Report No. PAE/PTh 14/87, 1987 (to be published).

${ }^{15}$ H. Bethe, Z. Phys. 71, 205 (1931); L. Hulthén, Ark. Mat. Astron. Fyz. 26A, No. 11 (1938).

${ }^{16}$ M. Takahashi, J. Phys. C 10, 1289 (1977).

${ }^{17}$ A. Luther and I. Peschel, Phys. Rev. B 12, 3908 (1975).

${ }^{18}$ M. Betsuyaku and I. Yokota, Phys. Rev. B 33, 6505 (1986).

${ }^{19}$ T. A. Kaplan, P. Horsch, and J. Borysowicz, Phys. Rev. B 35, 1877 (1987).

${ }^{20}$ J. E. Hirsch and D. J. Scalapino, Phys. Rev. B 27, 7169 (1983).

${ }^{21}$ They arise because one has to distinguish whether or not $\mathbf{f}_{1}$ and $\mathbf{f}_{2}$ belong to $\mathbf{f}_{3}, \ldots, \mathbf{f}_{m+2}$.

${ }^{22}$ This correlation is automatically included in the Ansatz of D. Baeriswyl, in Proceedings of the Sixth International Conference on Nonlinearity in Condensed Matter: Lessons from the Past and Prospects for the Future, Los Alamos, May, 1986, edited by A. R. Bishop et al., Springer Series in Solid-State Sciences Vol. 69 (Springer-Verlag, Berlin, 1987), which leads to an excellent ground-state energy for $U \rightarrow \infty$.

${ }^{23}$ E. H. Lieb and F. Y. Wu, Phys. Rev. Lett. 20, 1445 (1968). 